\title{
BMJ Open Changes in mental health problems and suicidal behaviour in students and their associations with COVID-19-related restrictions in Norway: a national repeated cross-sectional analysis
}

To cite: Sivertsen $B$, Knapstad M, Petrie K, et al. Changes in mental health problems and suicidal behaviour in students and their associations with COVID-19related restrictions in Norway: a national repeated crosssectional analysis. BMJ Open 2022;12:e057492. doi:10.1136/ bmjopen-2021-057492

- Prepublication history for this paper is available online. To view these files, please visit the journal online (http://dx.doi org/10.1136/bmjopen-2021057492).

Received 21 September 2021 Accepted 20 January 2022

Check for updates

(C) Author(s) (or their employer(s)) 2022. Re-use permitted under CC BY-NC. No commercial re-use. See rights and permissions. Published by BMJ.

For numbered affiliations see end of article.

Correspondence to Professor Børge Sivertsen; borge.sivertsen@fhi.no

\section{ABSTRACT}

Objective The COVID-19 pandemic has had a devastating impact on higher education, with the closure of student campuses. The aim of this study was to examine changes and prevalence of mental health problems, suicidal ideation and suicidal behaviour, and their associations with COVID-19-related restrictions.

Design, setting and participants As part of the SHoTstudy in Norway, 62498 students completed an online questionnaire $(65.6 \%$ women; response rate of $34.4 \%)$ in March 2021. Data were compared with previous waves, conducted in 2018, 2014 and 2010.

Primary and secondary outcome measures Mental health problems were assessed using the Hopkins Symptoms Checklist. Suicidal ideation, suicide attempts and non-suicidal self-harm (NSSH) were assessed with three items drawn from the Adult Psychiatric Morbidity Survey, and thoughts of NSSH were assessed with one item from the Child and Adolescent Self-Harm in Europe study.

Results There was a significant increase in mental health problems from 2010 to 2021, and especially from 2018 (men: 27\%/women: 45\%) to 2021 (men: 41\%/women: $62 \%, p<0.001)$. A similar pattern was also observed for suicidal thoughts. Unlike previous waves, there were large geographical differences in mental health problems in 2021, which mapped onto the different levels of COVID-19 cases and regional COVID-19-related restrictions. There was a significant negative dose-response association between days spent physically on campus and both mental health problems and indicators of suicide risk. We found the fewer days spent on campus in the last 2 weeks, the higher levels of mental health problems during the same time period. There was also an association between days on campus and a higher prevalence of suicidal thoughts, NSSH and suicide attempts in the last year.

Conclusion This study demonstrates a sharp increase and disturbing levels of mental health problems and suicide risk among students during the COVID-19 pandemic. Although causal conclusions cannot be drawn, the associations between closure of campuses and mental problems emphasise the importance of having access to campuses for student well-being.
Strengths and limitations of this study

- The SHOT study (Students' Health and Well-being Study) is a national student survey for higher education in Norway.

- The strengths of the study include the large and heterogeneous sample, and use of official data documenting the COVID-19 cases and COVID-19-related restrictions which pertain to the same timeframe as the latest self-report data collection.

- Limitations of the study include the relatively low response rates in SHoT studies $(23 \%-34 \%)$.

\section{INTRODUCTION}

The outbreak of the COVID-19 pandemic has had an overwhelming impact on all sections of society, including higher education institutions across the world. In response to the pandemic, many governments imposed restrictions including national or regional lockdowns. Colleges and universities were forced to reduce their activities due to the restrictions, or close down their campuses, and move teaching and assessment to digital platforms. These unprecedented changes to students' living and study conditions, alongside the many general restrictions imposed by the government, such as social distancing and stay at home orders, may have negatively impacted the students' mental health.

The studies looking at this issue rely largely on data from general population studies and the findings are inconclusive. In one of the few prospective studies of mental health during the COVID-19 pandemic, Pierce et al found that self-reported mental health problems in the UK general population increased significantly at the onset of the pandemic in April 2020, with the greatest increase among young adults. ${ }^{1}$ However, by October 2020, 
the mental health of most adults had returned to prepandemic levels. ${ }^{2}$ Another UK study during the first lockdown in Spring 2020 also found that suicidal ideation had increased, again especially among young adults. ${ }^{3}$ In contrast, a population-based study found that the level of mental disorders, suicidal ideation and suicide deaths remained stable in Norway during the first 6 months of the COVID-19 pandemic compared with pre-pandemic levels. ${ }^{4}$ A recent systematic review of general population data from eight countries showed high rates of mental health problems, although the lack of pre-pandemic data makes trend analyses difficult to interpret. ${ }^{5}$ Importantly, that review also identified students as one particularly at-risk subgroup for mental health problems. Students may be particularly vulnerable to social restrictions and social isolation, ${ }^{6}$ as they often live alone and are more likely to be single.

Indeed, recent meta-analyses of higher education students have found higher rates of anxiety and depressive symptoms during the first stage of the pandemic compared with pre-pandemic levels. ${ }^{78}$ Other early studies from the beginning of the pandemic also showed that during the first 5 weeks of 'lockdown' mental well-being decreased. ${ }^{9}$ Still, findings remain inconsistent, as a living systematic review of longitudinal studies does not suggest significant change in mental health among university students from before to during the COVID- $19 .{ }^{10}$ Similarly, a US study did not find elevated rates of suicidal ideation among 1754 university students in Fall 2020 versus two earlier semesters. ${ }^{11}$ However, the evidence base is largely focused on the first part of the pandemic, and the longstanding effects of restrictions over time have been less studied. A large French study found a high prevalence of mental health problems among students who experienced quarantine, ${ }^{12}$ and the rate of probable Post-traumatic stress disorder (PTSD) among university students remained high 1 month after living in quarantine during the national lockdown in France. ${ }^{13}$ However, there is a need for more studies to examine to what extent mental health problems could potentially be associated with different levels of imposed restrictions. For example, while educational institutions in some regions or countries practised complete campus lockdown, regions with fewer COVID-19 cases imposed less severe restrictions, enabling students to study on campus for some days each week. It remains unknown if such different levels of constraints might have an effect on the students' mental health. Also, all studies thus far have only reported data from the first few months of the pandemic, therefore little is known about the mental health of students 1 year after COVID-19 struck.

The aim of the present study, therefore, was to conduct a detailed investigation of the prevalence and changes in mental health problems, non-suicidal selfharm (NSSH) and suicide attempts among college and university students from pre-pandemic times to March 2021, and to examine associations with COVID-19related restrictions.

\section{METHODS}

\section{Study design, participants and setting}

The SHoT study (Students' Health and Well-being Study) is a national student survey for higher education in Norway, initiated by the three largest student welfare organisations. So far, four health surveys of the student population (aged 18-35) in Norway have been completed (2010, 2014, 2018 and 2021), and all four waves were collected electronically through a web-based platform. The four studies were conducted separately (not a longitudinal data collection). Details of the SHoT study have been published elsewhere. ${ }^{14}$ The SHoT2010 study was conducted between 11 October and 8 November 2010 . The target group was a random sample of 26779 Norwegian full-time students, of whom 6053 students completed the survey, yielding a response rate of 22.6\%. The SHoT2014 study was conducted between 24 February and 27 March 2014. An invitation email containing a link to an anonymous online questionnaire was sent to 47514 randomly selected students and stratified by study institutions, faculties and departments. The overall response rate was $28.5 \%$ and included 13525 students. The SHoT2018 was conducted between 6 February and 5 April 2018 inviting all full-time Norwegian students pursuing higher education (both in Norway and abroad). For the SHoT2018 study, 162512 students fulfilled the inclusion criteria, of whom 50054 students completed the online questionnaires, yielding a response rate of 30.8\%. The SHoT2021 was conducted between 1 March and 6 April 2021. This was a shorter health survey focusing specifically on mental health outcomes during the COVID-19 lockdown. In all, 181828 students were invited to participate, of which 62498 students completed the survey, yielding a response rate of 34.4\%. The SHoT2021 included students from all 32 universities and colleges. All parts of the project, including the planning of research questions, selection of study questionnaires, piloting, collection of data, as well as utilisation of data and findings, were conducted in close collaboration with the student welfare organisations in Norway, where student representatives were present. The email and SMS invitation for the SHoT2021 included a brief description of the study aim, which was to broadly examine students' health and well-being during the COVID-19 pandemic.

While the pandemic has had a major impact worldwide, countries have differed in their response and the nature and extent of restrictions imposed. In Norway, there has been a relatively low infection rate compared with other European countries. While there have not been any complete lockdowns or nationwide curfews, containment measures to restrict social contact, including the closure of, or limited access to, campuses and restrictions on many other services have been common in some regions. The present data collection was completed between 1 March and 6 April, and there were several levels of national and regional restrictions during this period, as detailed in figure 1 (panel B). Details of the various restriction levels can be found here. ${ }^{15}$ In the later part of the data 


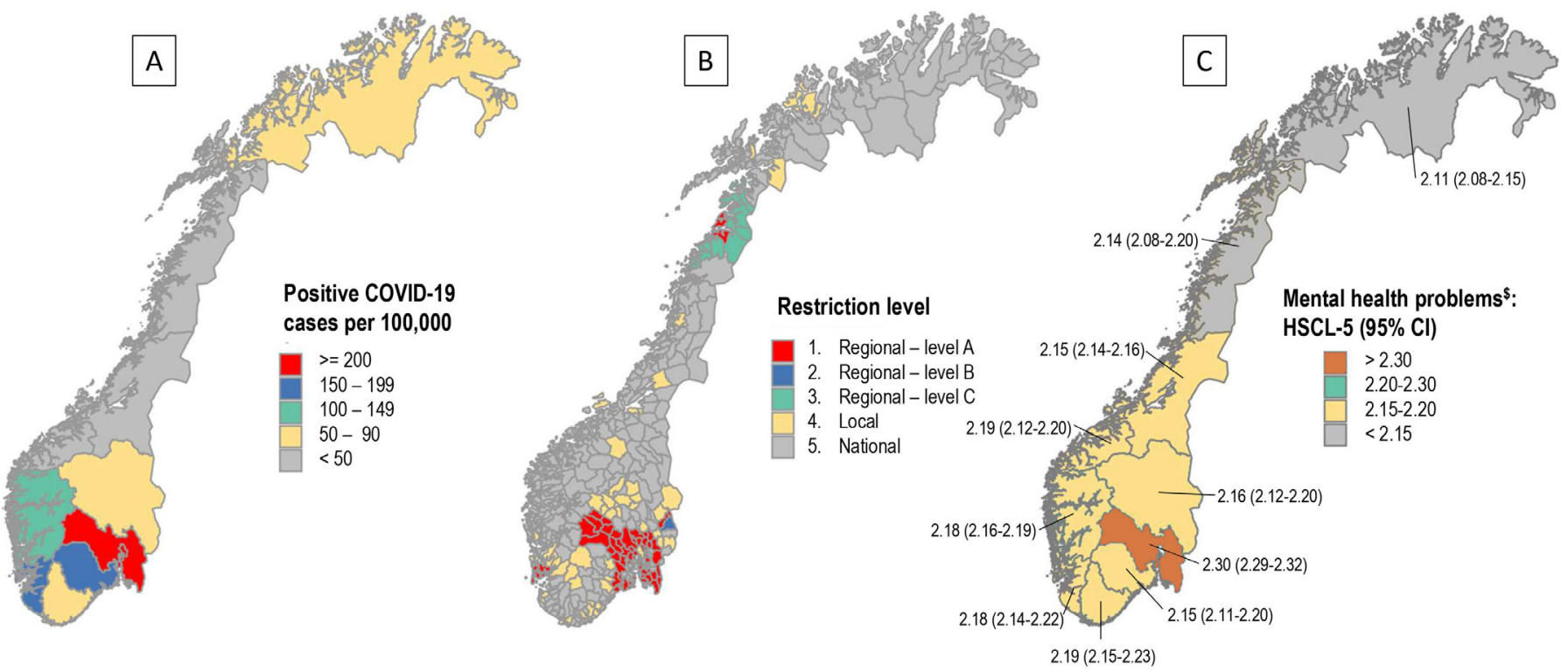

Figure 1 Geographical differences in number of positive COVID-19 cases (panel A), COVID-related restrictions (panel B) and mental health problems in the SHoT2021 study (HSCL-5; panel C). Data for all three figures are based on the situation in March (only) 2021. Sources: A-B: The Norwegian Institute of Public Health. \$ Estimated marginal means (EMM) of HSCL-5, adjusting for sociodemographic and COVID-19 factors (\# of tests, positive test, quarantine). HSCL, Hopkins Symptoms Checklist.

collection (from $25 \mathrm{March}),{ }^{16}$ the government imposed stricter national measures due to a rise in infection cases and worry about more contagious strains of the virus. Examples of these restrictions were the banning of all organised indoor sporting and recreational activities for adults, and for higher education the main rule was that all campuses were closed to students, and all teaching activities should be carried out digitally.

\section{Procedure and measures}

All students provided data on their age, gender, relationship status (single vs married/partner/boyfriend/ girlfriend) and parental education. Indication of gender had three possible response options: 'Woman', 'Man' and 'Other'. Financial difficulties were defined as participants, that during the last 12 months had experienced ('sometimes' or 'often') difficulties affording costs of living (such as for food, transportation and accommodation). Ethnicity was coded as Norwegian if the student or his/ her parents were born in Norway. Based on the geographical location of each educational institution, students were categorised according to Norway's recent county reform, which now includes 10 counties. All students were also asked how many days they had physically spent on campus during the last 14 days, due to COVID-19 restrictions. They also reported if they had been tested for COVID-19, number of tests, positive test (confirmed by an established test) and having been in quarantine.

Mental health problems were assessed using The Hopkins Symptoms Checklist (HSCL-5), ${ }^{17}$ derived from the 90-item Symptom Checklist, a screening tool designed to detect symptoms of anxiety and depression. The HSCL-5 is comprised of 5 items scored on a Likerttype scale from 1 ('not at all') to 4 ('extremely'). The reference period is the two last weeks. An average score on the HSCL-5 $>2.0$ is commonly used as a cut-off for identifying a high level of mental health problems, and corresponds well with the original cut-off of 1.75 on the longer version HSCL-25. ${ }^{18}$ Previous studies have shown very high correlations between the HSCL-5 and the widely used HSCL-25 ( $r>0.91) .{ }^{19}$ We have previously confirmed measurement invariance of HSCL-25 across time. ${ }^{20}$

History of suicidal thoughts, suicide attempts and NSSH were assessed with three items drawn from the Adult Psychiatric Morbidity Survey ${ }^{21}$; Have you ever seriously thought of taking your life, but not actually attempted to do so?', Have you ever made an attempt to take your life, by taking an overdose of tablets or in some other way?' and 'Have you ever deliberately harmed yourself in any way but not with the intention of killing yourself? (ie, self-harm)'. The question about NSSH thoughts was adapted from the Child and Adolescent Self-Harm in Europe study ${ }^{22}$; 'Have you ever seriously thought about trying to deliberately harm yourself but not with the intention of killing yourself, but not actually done so?'. If respondents answered yes to any item, the timing of the most recent episode was assessed, using the following response options: 'last week', 'past year', 'more than a year ago, but after I started studying at the university' and 'before I started studying at university'. In the current study, we defined 'recent' event as an episode having occurred within the past 12 months (the first two response options). In addition to the four self-injurious thoughts and behaviour questions asked in SHoT2018 and SHoT2021, suicidal thoughts were also measured by one item of the depression subscale of the HSCL- $25^{23}$ ('in the past 2 weeks, including today, how much have you 
been bothered by thoughts of ending your life') across all four SHoT study waves. The response options were 'not at all', 'a little', 'quite a bit' and 'extremely'.

\section{Statistical analyses}

IBM SPSS Statistics 27 for Windows (SPSS) was used for all statistical analyses. Pearson's $\chi^{2}$ tests were used to examine changes in the prevalence of mental health problems and suicidal-related factors. Estimated marginal means (EMMs) was computed using the UNIANOVA procedure in SPSS (which provides regression analysis and analysis of variance) to examine changes in continuous outcomes (HSCL-5), adjusting for age, sex and financial difficulties. Geographical differences in mental health problems (HSCL-5) were examined by computing EMMs, adjusting for sociodemographic factors (age, sex, ethnicity, financial difficulties and parental education), and COVID-19 factors (\# of tests, positive test, having been in quarantine). Logistic regression analysis, also adjusting for the same confounders was used to examine the association between days spend physically on campus, and mental health problems and suicide-related factors. There was generally very little missing data $(\mathrm{n}<140$ of 62498$)$ on the included variables, and the missing values were handled using listwise deletion.

\section{Patient and public involvement}

The planning and design of the study were initiated and governed by the student welfare organisations, which included deciding inclusion and exclusion criteria, and selecting potential research questions and instruments. Students were not involved in the actual collection of data, although recruitment was conducted in close collaboration with all the student welfare organisations in Norway. The results will be disseminated to the study participants via outlets of the student welfare organisations and educational institutions, with newsletters highlighting main findings being made available to all students. Popular summaries of results and interpretations with interest for a wider audience will be disseminated in appropriate outlets (eg, the web pages of educational institutions and the Norwegian Institute of Public Health (NIPH).

\section{RESULTS}

\section{Sample characteristics}

The SHoT2021 sample included 62498 young adults ( $65.6 \%$ women), with a mean age of 24.1 years $(\mathrm{SD}=5.2)$. In all, 225 students $(0.4 \%)$ identified as being transgender. About half $(50.8 \% \quad(n=31533))$ of the sample reported being single. In terms of ethnicity, $8.6 \%$ of the sample $(n=5365)$ were immigrants, defined as either the student or their parents being born outside Norway. The educational level of the students' parents was high, as detailed in table 1 . In terms of COVID-19, only $2.4 \%$ of the sample had tested positive for COVID-19, while 69.2\% had been tested. The majority of the sample (51.8\%) had
Table 1 Sociodemographic and clinical characteristics of the SHoT2021 study

\begin{tabular}{lc}
\hline & Total (n=62498) \\
\hline Age, mean (SD) & $24.1(5.2)$ \\
\hline Gender & $50.8(31533)$ \\
\hline Women & $65.6(40807)$ \\
\hline Men & $34.2(21405)$ \\
\hline Single, \% (n) & $50.8(31533)$ \\
\hline Ethnicity, \% (n) & \\
\hline Norwegian & $91.4(56847)$ \\
\hline Non-Norwegian & $8.6(5365)$ \\
\hline Maternal education, \% (n) & $5.1(3075)$ \\
\hline Primary & $28.9(17319)$ \\
\hline Secondary & $66.0(39531)$ \\
\hline College/university & \\
\hline Paternal education, \% (n) & $6.3(3721)$ \\
\hline Primary & $37.0(21714)$ \\
\hline Secondary & $56.7(33300)$ \\
\hline College/university & \\
\hline COVID-19 items & $61.8(32187)$ \\
\hline Tested, \% (n) & $2.4(1.8)$ \\
\hline Tested positive, \% (n) & \\
\hline Been in quarantine, \% (n) & \\
\hline
\end{tabular}

been in some form of quarantine, which typically entails 10 days of staying at home/avoiding social contact.

While demographical details of the earlier SHoT waves have been published elsewhere, table 2 shows that women have typically constituted around two-thirds of the participants in all surveys, although the 2018 survey had a slightly higher proportion of women. ${ }^{20}$ This differs a little from the gender distribution in higher education in Norway during the same period (around $60 \%$ women) ${ }^{24}$ As also detailed in table 2, fewer students reported often having financial difficulties in 2021 than in previous waves.

\section{Changes in mental health problems}

There was a significant increase in mental health problems from 2010 to 2021. Compared with the increase observed between earlier SHoT waves, ${ }^{20}$ the increase was notably stronger from 2018 to 2021 (figure 2). This pattern was evident both when examining the HSCL-5 categorically using predefined cut-offs (figure 2), as well as when using the measure as a continuous mean score (table 3). The trend was similar in both male and female students.

\section{Changes in suicidal thoughts, suicide attempts and NSSH}

We examined the prevalence of suicidal thoughts across all four SHoT studies from 2010 to 2021 (figure 3). There was a significant overall increase in students reporting suicidal thoughts the last 14 days ('a little', 'quite a bit' 
Table 2 Descriptive statistics of study participants in the four SHoT waves

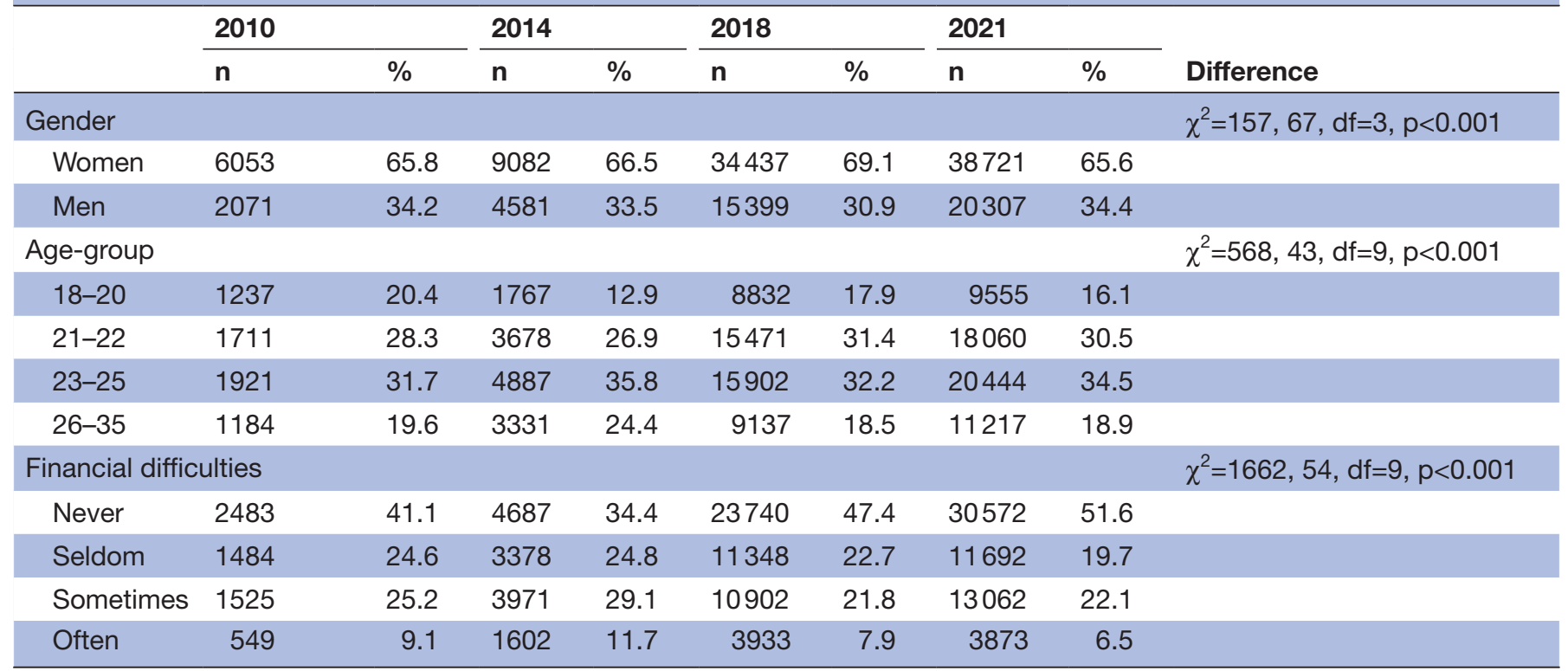

or 'extremely') from 2010 to 2021 in both male students (from $8.1 \%$ to $15.7 \%$ ) and female students (from $7.3 \%$ to $14.7 \%$ ). It is notable that the increase was also particularly strong from 2018 to 2021, especially among female students. The increase from 2018 to 2021 was largely driven by change in the 'a little' category, and not so much in the 'extremely' category.

While we had no data on suicide attempts and NSSH in 2010 and 2014, results from the last two waves (2018 and 2021) showed a gender-specific pattern: the prevalence of NSSH last year increased significantly for male students (from $2.1 \%$ to $2.4 \%, \mathrm{p}<0.05$ ), but not for female students (see figure 4 for details). The same pattern was observed for NSSH thoughts; while there was an increase in male students (from $4.5 \%$ to $5.0 \%, \mathrm{p}<0.05$ ), no change was observed for female students. There were no significant changes in last year suicide attempt. It should be noted the prevalences observed for male students, especially for

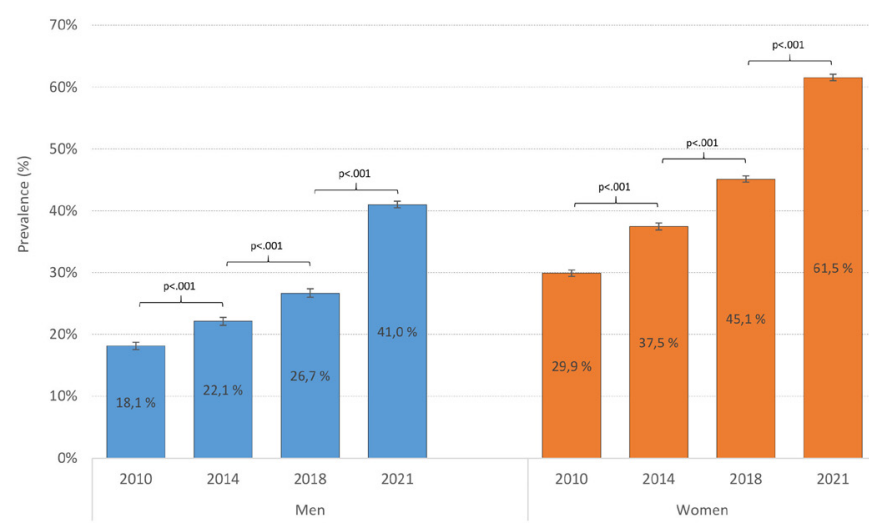

Figure 2 Trends in mental health problems from 2010 to 2021 in the SHoT study. Bars represent proportion of students scoring above the 2.0 cut-off on the HSCL-5. Error bars represent $95 \%$ Cl. HSCL, Hopkins Symptoms Checklist.
NSSH and NSSH thoughts, remained significantly lower than for female students.

\section{Geographical differences}

There were large geographical differences in COVID-19 cases, as displayed in figure 1; panel A. In March 2021, the South-Eastern region surrounding the capital of Oslo (marked in red) had substantially more COVID-19 cases compared with other areas in Norway. As displayed in figure 1; panel $\mathrm{B}$, there were also large geographical variations in terms of imposed national, regional and local COVID-19-related restrictions in March 2021. As expected, the strictest measures (marked in red) followed the same geographical distribution as the COVID-19 cases. Although the SHoT waves from 2010 to 2018 found only marginal or no geographical differences in mental health problems, ${ }^{25}$ the 2021 survey revealed significant geographical differences in adjusted mental health problems during the data collection in March 2021. As displayed in figure 1, students studying at an institution in South-Eastern region (marked in red) reported significantly more mental health problems compared with other geographical regions, after adjusting for sociodemographic-related and COVID-19-related factors.

\section{Days spent physically on campus, mental health problems, NSSH and suicidal thoughts and attempts}

We then examined the extent to which mental health problems were associated with the number of days physically spent on campus. As displayed in figure 5, there was a significant negative dose-response association between mental health problems and days spent on campus. Students spending 7+ days on campus the last 2 weeks, reported significantly fewer mental health problems during this period, compared with students 
Table 3 Mental health problems (HSCL-5) in the four SHoT waves

\begin{tabular}{|c|c|c|c|c|c|c|c|c|c|}
\hline & \multicolumn{2}{|c|}{$\mathrm{T} 1-2010$} & \multicolumn{2}{|c|}{ T2-2014 } & \multicolumn{2}{|c|}{ T3-2018 } & \multicolumn{2}{|c|}{ T4-2021 } & \multirow[b]{2}{*}{ Time points differences } \\
\hline & Mean & SD & Mean & SD & Mean & SD & Mean & SD & \\
\hline Total sample & 1.75 & 0.69 & 1.87 & 0.71 & 2.00 & 0.79 & 2.27 & 0.78 & $\mathrm{~T} 1<\mathrm{T} 2<\mathrm{T} 3<\mathrm{T} 4^{*}$ \\
\hline Men & 1.57 & 0.64 & 1.68 & 0.65 & 1.74 & 0.72 & 2.02 & 0.74 & $\mathrm{~T} 1<\mathrm{T} 2<\mathrm{T} 3<\mathrm{T} 4^{*}$ \\
\hline
\end{tabular}

${ }^{*}$ All differences significant at $\mathrm{p}<0.001$.

HSCL, Hopkins Symptoms Checklist.

who were not permitted on campus, after adjusting for sociodemographic-related and COVID-19-related factors.

A similar inverse dose-response association was observed between days spent on campus and recent NSSH, suicidal thoughts and suicide attempts. For example, as detailed in figure 6 and table 4, the prevalence of self-harm in the last year was $4.2 \%$ among students who spent no days on campus in the last 2 weeks, compared with $2.9 \%$ among students who spent $7+$ days on campus (adj. OR=1.44, $95 \%$ CI 1.25 to 1.67$)$. Similar significant graded associations were observed also for the other three self-harm and suicide-related items. For sensitivity purposes, we also conducted the above analyses using self-harm/suiciderelated items in the last week as the dependent variable (data not shown). The same pattern emerged for these analyses; clear inverse dose-response association (with the exception of actual suicide attempt last week, due to lack of statistical power/few overall events; $n=31$ ).

\section{DISCUSSION}

Using data from the most recent wave of national Students' Health and Well-being Study collected about 12 months into the COVID-19 pandemic, it is clear that there were substantial increases in both mental health problems and suicidal thoughts from 2010 to 2021, and especially from 2018 to 2021. There were also large geographical differences in the prevalence of mental health problems in 2021, which corresponded closely to both levels of COVID-19 cases in these areas of Norway and the regionally dependent COVID-19 restrictions.

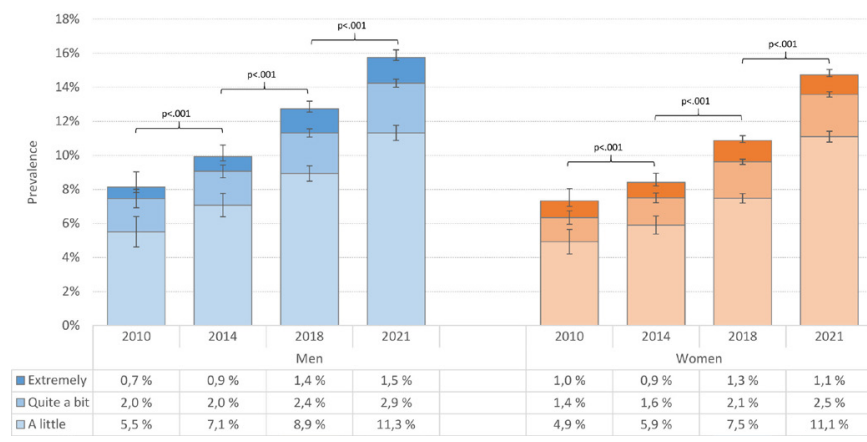

Figure 3 Trends in suicidal thoughts (past 2 weeks) from 2010 to 2021 in the SHoT study. Bars represent responses on the HSCL-25 suicidal thoughts item, while error bars represent 95\% Cls. HSCL, Hopkins Symptoms Checklist.
Such geographic differences have not been seen in the previous study waves. We also found significant negative dose-response associations between days spent physically on campus and both mental health problems and suiciderelated variables; the fewer days being present on campus the last 2 weeks, the higher level of mental health problems during the same time period and also higher prevalence of suicidal thoughts, suicide attempts and NSSH in the last year.

The current study provides new evidence about the mental health status of college and university students 1 year after COVID-19 struck, and how studying under prolonged restrictions may affect the lives of young adults pursuing higher education. Our findings differ from previous studies of the general population, which showed improvements in mental health problems following an initial deterioration at the beginning of the pandemic. Most of these studies reported on mental health trajectories during the first few weeks and months following the outbreak, concluding that a swift increase in mental health problems in the early stages of the pandemic was quickly followed by a similar decline in symptoms of anxiety and depression. ${ }^{6}$ A slightly different picture was provided by Pierce $e t$ al who found that the mental health

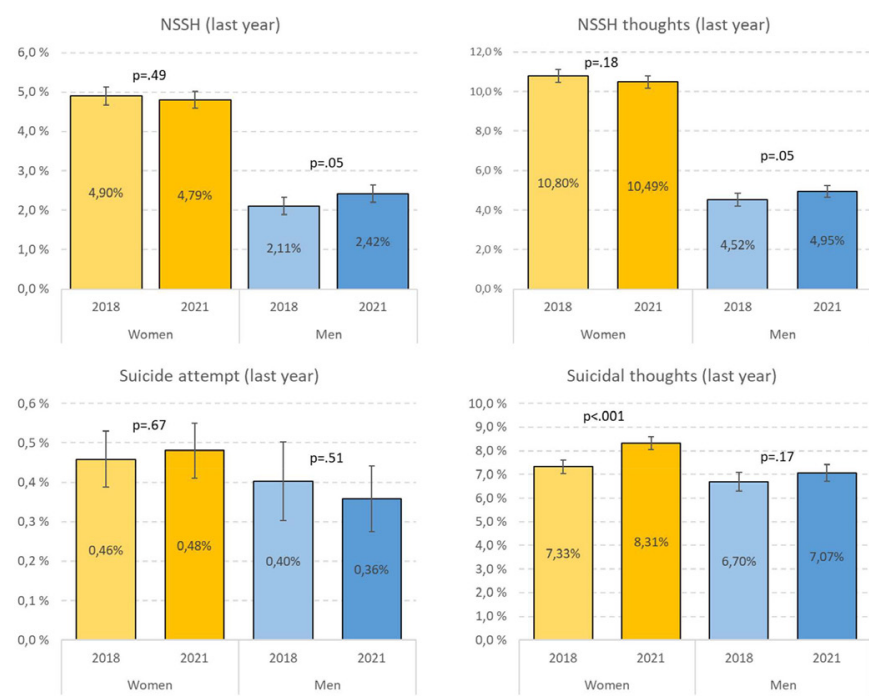

Figure 4 Trends in suicidal thoughts, suicide attempts and non-suicidal self-harm reported in the last year from 2018 to 2021 in the SHoT study. Error bars represent 95\% Cls; p values are based on $\chi^{2}$ tests. NSSH, non-suicidal self-harm. 


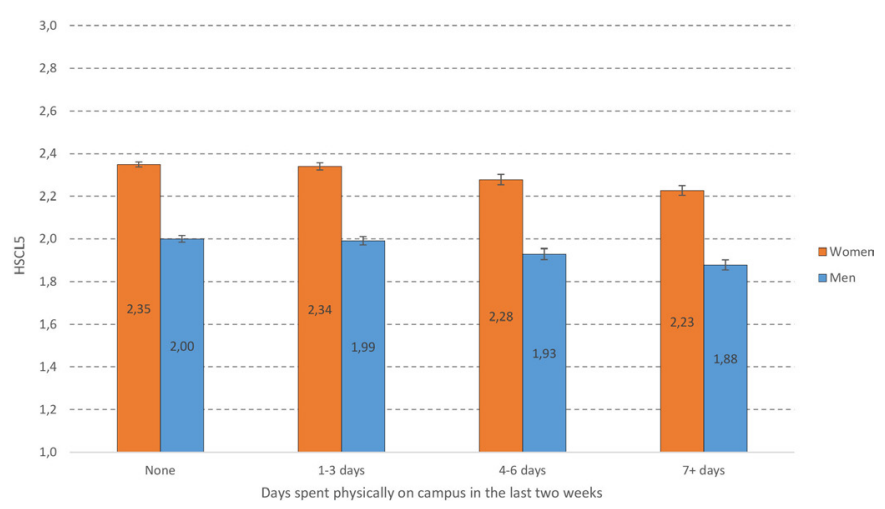

Figure 5 Mental health problems (EMM of HSCL-5 mean score) by days spent physically on campus in the last 2 weeks, adjusting for sociodemographic and COVID-19 factors (\# of tests, positive test and quarantine). EMM, estimated marginal mean; HSCL, Hopkins Symptoms Checklist.

of the general population steadily improved through to October 2020, but not to pre-pandemic levels. ${ }^{26}$ Of note, the latter study also identified vulnerable subgroups who did not experience the overall improvements in mental health, but rather reported a steadily worsening to levels far worse than before the pandemic. Interestingly, being young was associated both with having a recovering and with a deteriorating trajectory. Other studies have also shown that college students and young people in general are particularly vulnerable for developing ill mental health during the pandemic, ${ }^{3578}$ and the current study does indeed suggest this to be the case. The vulnerability could both be in terms of being in a developmental period with many changes, and including establishing new social networks and social roles. Common spaces including campuses, recreational facilities and cafes and pubs may be more important venues for social contact for students than both younger and older people, and thus the restrictions may have a larger impact on their lives. Students may also have been vulnerable due to the many

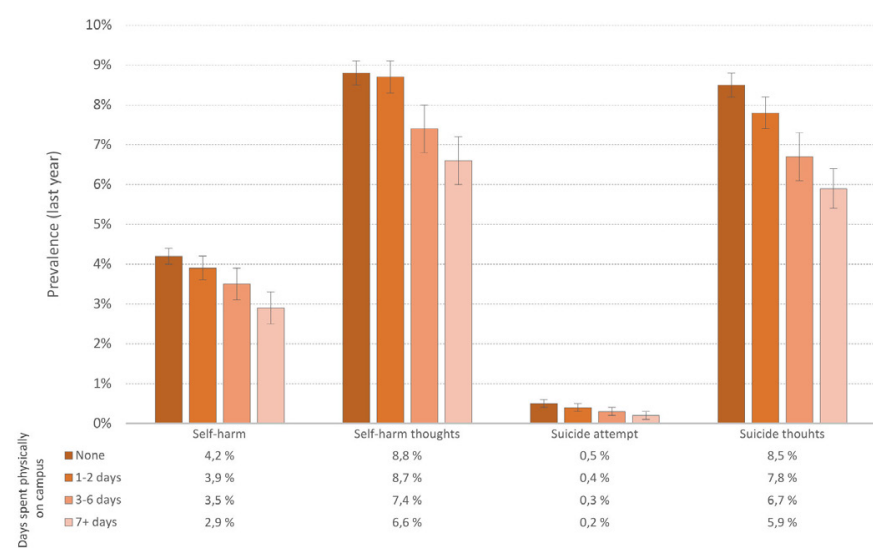

Figure 6 Estimated prevalence of recent (last year) nonsuicidal self-harm, suicidal thoughts and suicide attempts by days spent physically on campus in the last 2 weeks, adjusting for sociodemographic and COVID-19 factors (\# of tests, positive test, quarantine). restrictions specifically targeting students in Norway. While our dataset did not include multiple assessments during the pandemic and selection bias cannot be precluded, it does show a disturbingly sharp deterioration of students' mental health over time, especially from 2018 to 2021 . While this may be partly a result of a pattern of worsening self-reported mental health among students the last years ${ }^{20}$ we do not consider it likely that this could account for the sudden sharp drop in mental health and increase in suicidal thoughts over just 3 years.

Another novel and important finding of the current study was the graded associations between days spent physically on campus and both mental health problems and suicide-related variables. As the imposed COVID-19 restrictions in Norway varied greatly during the data collection in March 2021, we were able to examine how regional differences in access to campuses coincided with mental health problems during the same time. While previous data waves of the SHoT study have found quite similar levels of mental health problems across all geographical regions, the current study found that students in some regions reported substantially worse mental health than others. The region around Oslo had significantly more mental health problems compared with the rest of the country-and that region also had by far the most COVID-19 cases and strongest restrictions at that time, included limited access to college and university campuses. As such, the current study extends on the two French studies by Wathelet and colleagues, who found living under quarantine was associated with high rates if mental health problems and probable PTSD among students. Combined with the current study, these data stress the importance of access to campuses for student well-being. It should also be noted that studies from the general population have found lower levels of mental health problems during the COVID-19 outbreak in Norway compared with other countries. ${ }^{27}$ As such, mental health outcomes in countries with a more severe COVID-19 situation and stricter lockdown measures may be even worse.

Our findings have some important clinical and public health implications. The steep increase and high levels of mental health problems and suicide-related factors observed 1 year into the pandemic emphasise the need for immediate attention to students pursuing higher education as a vulnerable group. Both policy makers, educational institutions and student welfare organisations need to ensure that the necessary support mechanisms are in place, to maximise preparedness for similar events in the future. Although the pandemic may have forced a widespread adoption of digital platforms to provide both teaching and assessments, there is an urgent need to scale up the provision of digital mental health services in addition to existing facilities available on campuses. Moreover, our data suggest that the swift and widespread implementation of digital platforms to replace human interactions with teachers and fellow students should be done with caution, as staff readiness, student accessibility, 
Table 4 OR for poor mental health (HSCL-5 >2) and suicidal history factors by days spent physically on campus in the last 2 weeks, adjusting for sociodemographic and COVID-19 factors (\# of tests, positive test, quarantine)

\begin{tabular}{|c|c|c|c|c|c|c|c|c|}
\hline & \multicolumn{8}{|c|}{ Days spent physically on campus in the last 2 weeks } \\
\hline & \multicolumn{2}{|l|}{ None } & \multicolumn{2}{|c|}{ 1+3 days } & \multicolumn{2}{|c|}{ 4-6 days } & \multicolumn{2}{|c|}{$7+$ days } \\
\hline & OR & $95 \% \mathrm{Cl}$ & OR & $95 \% \mathrm{Cl}$ & OR & $95 \% \mathrm{Cl}$ & OR & $95 \% \mathrm{Cl}$ \\
\hline \multicolumn{9}{|c|}{ Poor mental health (HSCL-5 >2) } \\
\hline Total sample & 1.36 & 1.29 to 1.43 & 1.30 & 1.22 to 1.37 & 1.19 & 1.11 to 1.27 & 1.00 & - \\
\hline Women & 1.35 & 1.26 to 1.45 & 1.33 & 1.23 to 1.43 & 1.22 & 1.12 to 1.33 & 1.00 & - \\
\hline \multicolumn{9}{|c|}{ Suicidal history factors (total sample) } \\
\hline Self-harm & 1.44 & 1.25 to 1.67 & 1.35 & 1.15 to 1.58 & 1.24 & 1.03 to 1.49 & 1.00 & - \\
\hline Self-harm thoughts & 1.30 & 1.18 to 1.44 & 1.31 & 1.18 to 1.46 & 1.10 & 0.98 to 1.25 & 1.00 & - \\
\hline Suicide attempts & 2.15 & 1.18 to 1.46 & 1.95 & 1.13 to 3.35 & 1.41 & 0.74 to 2.68 & 1.00 & - \\
\hline Suicidal thoughts & 1.42 & 0.98 to 1.25 & 1.33 & 1.19 to 1.49 & 1.14 & 1.00 to 1.30 & 1.00 & - \\
\hline
\end{tabular}

HSCL, Hopkins Symptoms Checklist.

social meeting points and motivation are important parts of the learning process, both digital and traditional. ${ }^{28}$ And while stringent restrictions may be a necessary evil during the peak of a pandemic, prolonged lockdown of student campuses should be kept to a minimum, where possible.

Strengths of the current study include the large and heterogeneous sample, and use of official data documenting the COVID-19 cases and COVID-19-related restrictions which pertain to the same timeframe as the SHoT data collection. The most important study limitation is the cross-sectional design of the SHoT2021, which limits our ability to examine the directionality between days spent on campus and mental health problems and suicide-related variables. As such, we cannot disregard the possibility of the students with existing mental health problems will spend fewer days on campus, compared with students without such problems. Second, the study did not include multiple assessments points neither prior to or during the pandemic, and as such we were unable to explore detailed trajectories of the students' mental health from pre-pandemic to during, and during the first weeks and months of the pandemic. Related to this, we cannot disregard the possibility that the increase in the number of students attending college and university from over time may have influenced the results. Third, the attendance rate was relatively modest $(34.4 \%)$, with no information about non-participants other than the age and gender distribution. We neither know to what extent non-participation patterns were similar across study waves. While it has been shown that non-participants of health surveys generally have worse health than participants, ${ }^{29}$ people may also be more prone to participate in a survey if the topic seems relevant to them personally. ${ }^{30}$ In the communication material used in the SHoT studies, it is emphasised that we want to assess 'how the students really are and feel', and as such, one may speculate if this phrasing may lead to a higher participation rate of individuals who feel that the topic is of particular relevance to them.

Forth, updated optimal cut-offs for HSCL-5 in general, and for student populations in particular, are lacking. Hence, more emphasis should be given to changes in the continuous than the dichotomous HSCL-5 scores. Fifth, assessment of mental health problems and the suiciderelated variables were based on self-report, and consequently are subject to demand characteristics. However, this latter limitation is unlikely to explain any of the main findings of this study. Finally, we did not standardise the data collection procedures across sites, with some (but not all), universities allowing their students to fill out the questionnaire during lectures.

To conclude, the current study provides evidence of a sharp increase and disturbingly high levels of mental health problems and suicide-related factors among students pursuing higher education during the COVID-19 pandemic. Although causal conclusions cannot be drawn, the current study may indicate that the COVID-19 pandemic has negatively impacted on students' mental health. The close link between limited access to college campuses and mental ill-health emphasises the negative effects of social isolation during prolonged campus lockdown.

\section{Author affiliations}

${ }^{1}$ Department of Health Promotion, Norwegian Institute of Public Health, Bergen, Norway

${ }^{2}$ Department of Mental Health, Norwegian University of Science and Technology, Trondheim, Norway

${ }^{3}$ Department of Research \& Innovation, Helse Fonna HF, Haugesund, Norway ${ }^{4}$ Department of Psychological Medicine, University of Auckland, Auckland, New Zealand

${ }^{5}$ Suicidal Behaviour Research Laboratory, Institute of Health \& Wellbeing, University of Glasgow, Glasgow, UK

${ }^{6}$ The Student Welfare Organization in Oslo and Akershus (Si0), Oslo, Norway

${ }^{7}$ The Norwegian Medical Association, 0slo, Norway 
${ }^{8}$ Department of Psychosocial Science, University of Bergen, Bergen, Norway

\section{Twitter Keith Petrie @keithpetrie}

Acknowledgements We wish to thank all participating students as well as the three largest student associations in Norway (SiO, Sammen and SiT), who initiated and designed SHoT studies. We also wish to thank the Library at the Norwegian Institute of Public Health for helping us with the literature search to the present study. A special thanks to Truc Trung Nguyen at NIPH for helping produce the graphical illustrations in Figure 1.

Contributors BS is the guarantor of the study. BS and KJL contributed with initiation, planning and design of the survey data collection. BS and MH planned, designed and coordinated the present study. BS conducted the statistical analyses on the survey data, conducted the literature review and led the writing of the manuscript. All authors, including KJL, MH, MK, RO, KP contributed with input on design and analytical plan, interpretation of results, writing of the first draft and critical revision of the manuscript and analyses. All authors approved the submission.

Funding SHoT 2018 has received funding from the Norwegian Ministry of Education and Research (2017) and the Norwegian Ministry of Health and Care Services (2016).

Map disclaimer The inclusion of any map (including the depiction of any boundaries therein), or of any geographic or locational reference, does not imply the expression of any opinion whatsoever on the part of BMJ concerning the legal status of any country, territory, jurisdiction or area or of its authorities. Any such expression remains solely that of the relevant source and is not endorsed by BMJ. Maps are provided without any warranty of any kind, either express or implied.

Competing interests None declared.

Patient and public involvement Patients and/or the public were involved in the design, or conduct, or reporting, or dissemination plans of this research. Refer to the Methods section for further details.

\section{Patient consent for publication Consent obtained directly from patient(s)}

Ethics approval This study involves human participants and was approved. All procedures involving human subjects/patients were approved by the Regional Committee for Medical and Health Research Ethics in Western Norway (SHoT2018: no. 2017/1176 and SHoT2021: no. 176205, respectively). Approvals for conducting the SHoT2010 and SHoT2014 studies were granted by the Data Protection Officer for research at the Norwegian Centre for Research Data. Electronic informed consent was obtained after complete description of the study to the participants. Participants gave informed consent to participate in the study before taking part.

Provenance and peer review Not commissioned; externally peer reviewed.

Data availability statement Data are available upon reasonable request. Al SHoT data set is administrated by the NIPH. Approval from a Norwegian regional committee for medical and health research ethics (https://helseforskning.etikkom. no) is a prerequirement.

Open access This is an open access article distributed in accordance with the Creative Commons Attribution Non Commercial (CC BY-NC 4.0) license, which permits others to distribute, remix, adapt, build upon this work non-commercially, and license their derivative works on different terms, provided the original work is properly cited, appropriate credit is given, any changes made indicated, and the use is non-commercial. See: http://creativecommons.org/licenses/by-nc/4.0/.

ORCID iDs

Børge Sivertsen http://orcid.org/0000-0003-4654-9296

Keith Petrie http://orcid.org/0000-0002-6337-2480

\section{REFERENCES}

1 Pierce M, McManus S, Hope $\mathrm{H}$, et al. Mental health responses to the COVID-19 pandemic: a latent class trajectory analysis using longitudinal UK data. Lancet Psychiatry 2021;8:610-9.

2 Pierce M, Hope H, Ford T, et al. Mental health before and during the COVID-19 pandemic: a longitudinal probability sample survey of the UK population. Lancet Psychiatry 2020;7:883-92.

3 O'Connor RCet al. Mental health and well-being during the COVID-19 pandemic: longitudinal analyses of adults in the UK COVID-19 Mental Health \& Wellbeing study. Br J Psychiatry 2020:1-8.

4 Knudsen AKS, Stene-Larsen K, Gustavson K, et al. Prevalence of mental disorders, suicidal ideation and suicides in the general population before and during the COVID-19 pandemic in Norway: a population-based repeated cross-sectional analysis. Lancet Reg Health Eur 2021;4:100071

5 Xiong J, Lipsitz O, Nasri F, et al. Impact of COVID-19 pandemic on mental health in the general population: a systematic review. J Affect Disord 2020;277:55-64

6 Fancourt D, Steptoe A, Bu F. Trajectories of anxiety and depressive symptoms during enforced isolation due to COVID-19 in England: a longitudinal observational study. Lancet Psychiatry 2021;8:141-9.

7 Chang J-J, Ji Y, Li Y-H, et al. Prevalence of anxiety symptom and depressive symptom among college students during COVID-19 pandemic: a meta-analysis. J Affect Disord 2021;292:242-54.

8 Deng J, Zhou F, Hou W, et al. The prevalence of depressive symptoms, anxiety symptoms and sleep disturbance in higher education students during the COVID-19 pandemic: a systematic review and meta-analysis. Psychiatry Res 2021;301:113863.

9 Savage MJ, James R, Magistro D, et al. Mental health and movement behaviour during the COVID-19 pandemic in UK university students: prospective cohort study. Ment Health Phys Act 2020;19:100357.

10 Sun Yet al. Comparison of mental health symptoms prior to and during COVID-19: evidence from a living systematic review and meta-analysis. medRxiv 2021

11 Gratz KL, Mann AJD, Tull MT. Suicidal ideation among university students during the COVID-19 pandemic: identifying at-risk subgroups. Psychiatry Res 2021;302:114034.

12 Wathelet M, Duhem S, Vaiva G, et al. Factors associated with mental health disorders among university students in France confined during the COVID-19 pandemic. JAMA Netw Open 2020;3:e2025591.

13 Wathelet M, Fovet T, Jousset A, et al. Prevalence of and factors associated with post-traumatic stress disorder among French university students 1 month after the COVID-19 lockdown. TransI Psychiatry 2021;11:327

14 Sivertsen B, Råkil H, Munkvik E, et al. Cohort profile: the SHoT-study, a national health and well-being survey of Norwegian university students. BMJ Open 2019;9:e025200.

15 The Norwegian Government. Levels of measures in the event of local outbreaks of infection, 2021.

16 The Ministry of Health and Care Services. The government is implementing stricter national measures, 2021.

17 Derogatis LR, Lipman RS, Rickels K, et al. The Hopkins symptom checklist (HSCL): a self-report symptom inventory. Behav Sci 1974;19:1-15.

18 Winokur A, Winokur DF, Rickels K, et al. Symptoms of emotional distress in a family planning service: stability over a four-week period. Br J Psychiatry 1984;144:395-9.

19 Strand BH, Dalgard OS, Tambs K, et al. Measuring the mental health status of the Norwegian population: a comparison of the instruments SCL-25, SCL-10, SCL-5 and MHI-5 (SF-36). Nord J Psychiatry 2003:57:113-8

20 Knapstad M, Sivertsen B, Knudsen AK, et al. Trends in self-reported psychological distress among college and university students from 2010 to 2018. Psychol Med 2021;51:1-9.

21 McManus Set al. Mental health and wellbeing in England: adult psychiatric morbidity survey 2014. NHS Digital: Leeds, 2016.

22 Madge N, Hewitt A, Hawton K, et al. Deliberate self-harm within an international community sample of young people: comparative findings from the Child \& Adolescent Self-harm in Europe (CASE) Study. J Child Psychol Psychiatry 2008;49:667-77.

23 Derogatis LR, Lipman RS, Rickels K, et al. The Hopkins symptom checklist (HSCL): a self-report symptom inventory. Behav Sci 1974;19:1-15.

24 Statistics Norway Studenter i høyere utdanning [Students taking higher education in Norway]. 2021.

25 Knapstad M. Sivertsen Studentenes helse- og trivelsundersøkelse 20182018.

26 Saunders R, Buckman JEJ, Fonagy P, et al. Understanding different trajectories of mental health across the general population during the COVID-19 pandemic. Psychol Med 2021:1-9.

27 Geirdal Amy Østertun, Ruffolo M, Leung J, et al. Mental health, quality of life, wellbeing, loneliness and use of social media in a time of social distancing during the COVID-19 outbreak. A cross-country comparative study. J Ment Health 2021;30:148-55.

28 Ali W. Online and remote learning in higher education Institutes: a necessity in light of COVID-19 pandemic. Higher Education Studies 2020;10:16-24.

29 Knudsen AK, Hotopf M, Skogen JC, et al. The health status of nonparticipants in a population-based health study: the Hordaland health study. Am J Epidemiol 2010;172:1306-14

30 Edwards PJ, Roberts I, Clarke MJ, et al. Methods to increase response to postal and electronic questionnaires. Cochrane Database Syst Rev 2009;3:MR000008. 